\title{
Impact of bronchiolitis guidelines publication on primary care prescriptions in the Italian pediatric population
}

\author{
Elisa Barbieri $\mathbb{D}^{1 凶}$, Anna Cantarutti $\mathbb{D}^{2}$, Sara Cavagnis ${ }^{1}$, Luigi Cantarutti ${ }^{3}$, Eugenio Baraldi ${ }^{4}$, Carlo Giaquinto ${ }^{1}$ and Daniele Donà ${ }^{1}$
}

In Italy, two clinical practice guidelines for the diagnosis and treatment of bronchiolitis were published in October 2014 and December 2015. We evaluated prescriptions for bronchiolitis in children aged 0-24 months before (December 2012-December 2014), in between (December 2014-December 2015) and after (December 2015-December 2018) the guidelines publications. Data were retrieved from the Pedianet database; the measured outcomes were prescriptions rates of antibiotics, corticosteroids, $\beta 2-$ agonists, and other respiratory drugs. In 1011 out of 1581 episodes, patients received at least one treatment, with a total of 2003 prescriptions. The rate of treated bronchiolitis decreased from $66 \%$ to $57 \%(p<0.001)$ after the publication of the second guideline; the highest reduction was in younger patients (from $57 \%$ to $44 \%, p=0.013$ ). Overall antibiotic prescriptions rate did not change, with $31.6 \%$ of the patients still receiving them. Our results confirm unnecessary non-evidence-based treatments in the primary care setting, with few changes after the guidelines publications.

npj Primary Care Respiratory Medicine (2021)31:15; https://doi.org/10.1038/s41533-021-00228-w

\section{INTRODUCTION}

Viral bronchiolitis is the most common lower respiratory tract infection in infants $^{1}$ and has a heavy impact on pediatric healthcare ${ }^{2,3}$.

Bronchiolitis is mainly caused by Respiratory Syncytial Virus $(\mathrm{RSV})^{4}$ and the diagnosis is based on medical history and clinical findings. Bronchiolitis is a self-limiting disease and lacks a specific etiological treatment, therefore management should be focused on supportive care, based on oxygen therapy and fluid supplementation $^{5-7}$. Pharmacological treatment with bronchodilators, corticosteroids, antibiotics, and other medications has been proven to be not useful in reducing symptoms severity or duration $^{8-12}$ and is currently not recommended in national and international guidelines ${ }^{5-7,13,14}$. In the Italian context, two guidelines were published: the first was published in 2014 by Baraldi et al., and was the result of the cooperation of 20 Italian pediatric scientific societies ${ }^{7}$. The second guideline is the Italian translation of the NICE guidelines by Cartabellotta et al., published in $2015^{13}$ and later recognized by the Italian guidelines society ("Società Nazionale Linee Guida") of the Italian Institute of Health ("Istituto Superiore di Sanità") as Good Clinical Practices.

The two guidelines have some minor differences in the diagnosis, management, and treatment of children with bronchiolitis. For instance, the document by Baraldi et al. refers to children up to 12 months, while the article by Cartabellotta et al., even though the Authors state that bronchiolitis is more common in the first year of life, considers children up to 2 years of age. Furthermore, when addressing the factors that should be considered when referring the child to secondary care, Baraldi et al. consider prematurity as $\mathrm{GA}<37$ weeks, while Cartabellotta et al. recommend the threshold of 32 weeks of GA. For what concerns the treatment of bronchiolitis, the second guideline is more strict in not recommending pharmacological therapies, while Baraldi et al. are less rigorous, allowing some exceptions, like a trial of inhaled salbutamol.
Despite these minor differences, both guidelines agree on the scarce role of pharmacological therapy because of a lack of evidence in treatment efficacy.

Unnecessary non-evidence-based treatment prescription is still very common, both in primary care, emergency department (ED) and hospital settings ${ }^{15-20}$; moreover, there is still considerable variability in diagnostic test utilization and disease management, potentially generating unnecessary and costly resources use $^{15,21,22}$.

With this study, we aimed to evaluate the impact of both guidelines on clinical practice in the pediatric primary care (PPC) setting, by comparing prescriptions of antibiotics, corticosteroids, and bronchodilators before and after guidelines publication.

\section{RESULTS}

Complete episodes selection is described in Fig. 1.

\section{Population characteristics}

The demographic characteristics of included patients are described in Table 1.

Of the 1581 episodes included in the study, 454 (28.7\%) patients were $0-3$ months old, 534 (33.8\%) were 4-6 months old and 593 (37.5\%) were 7-24 months old at the time of first visits.

$903(57.1 \%)$ patients were males, and the mean age at the time of first bronchiolitis visits was around 6 months and did not differ significantly between the three periods. The majority of the patients did not have follow-up visits $(84.1 \%)$ and seemed to be more frequent in younger children (20\%) compared to children aged $4-6$ months $(15.5 \%)$ or more $(12.8 \%)$.

The patients were mainly from Northern Italy (65.2\%), with no changes between periods.

\footnotetext{
'Division of Paediatric Infectious Diseases, Department of Women's and Children's Health, University of Padova, Padua, Italy. ${ }^{2}$ Department of Statistics and Quantitative Methods, Unit of Biostatistics Epidemiology and Public Health, University of Milano-Bicocca, Milan, Italy. ${ }^{3}$ Pedianet Project, Padua, Italy. ${ }^{4}$ Unit of Neonatal Intensive Care, Department of Woman's and Child's Health, University of Padova, Padua, Italy. ${ }^{凶}$ email: elisa.barbieri@unipd.it
} 


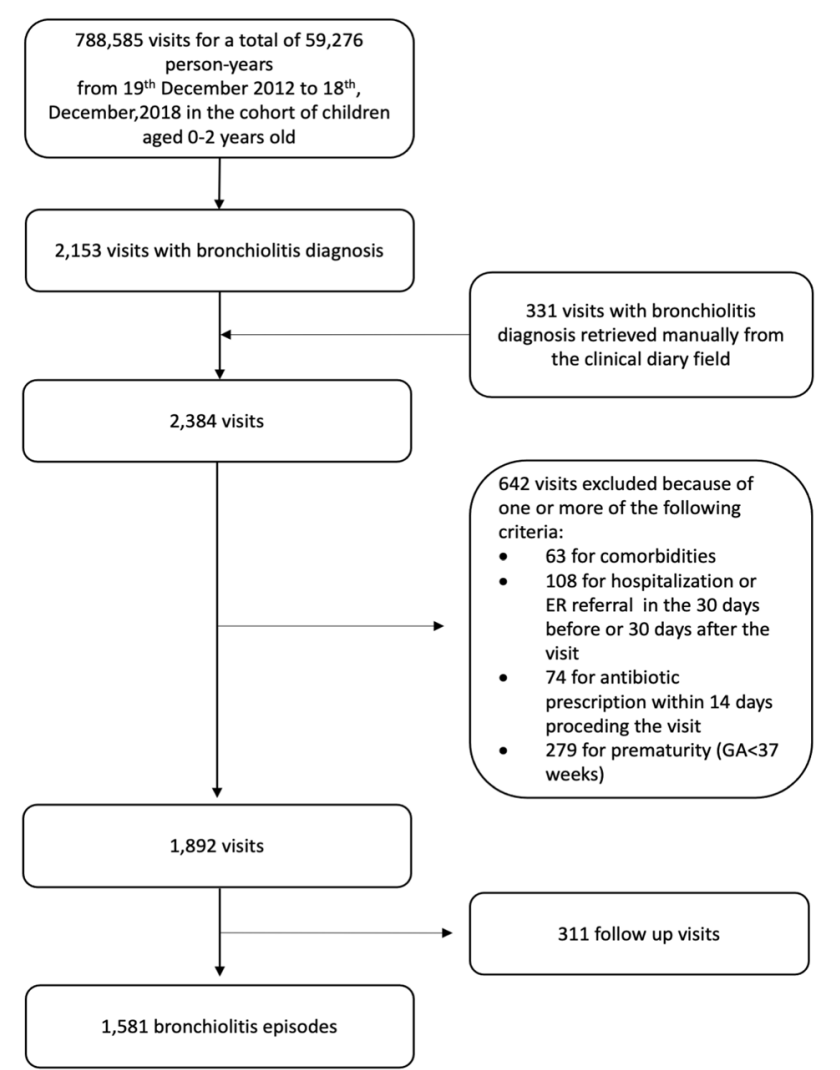

Fig. 1 Bronchiolitis episodes' inclusion flow chart (Pedianet, 2012-2018). ER emergency room, GA gestational age.

\section{Outcomes}

In 1011 out of 1581 episodes included in the study, patients received at least one treatment, with a total of 2003 prescriptions.

The number of bronchiolitis treated decreased from $66.2 \%$ to $56.6 \%$ in the Pre vs Post2 period ( $x^{2}$ test $p<0.001$ ). The highest decrease was noted in children younger than 3 months (from $57.0 \%$ to $43.7 \%$, $x^{2}$ test $-=0.013$ ), while children older than 7 months did not have a significant reduction (from $70.5 \%$ to $64.7 \%, x^{2}$ test $\left.p=0.198\right)$. Complete data are shown in Supplementary Table 1.

The total number of medicines prescribed decreased significantly after the publication of the two guidelines, but still in the $56.5 \%$ of the cases in the Post 2 period was prescribed at least one medicine and in the $12.7 \%$ three or more. (Supplementary Table 1).

In total, antibiotics were prescribed in 537 episodes; in the $33.5 \%$ of episodes in the Pre period at least one antibiotic was prescribed, compared to the $41.1 \%$ and the $31.6 \%$ in the Post 1 and Post2 periods respectively (Fig. 2a). Amoxicillin prescription rates increased from $11.1 \%$ to $15.6 \%$ in the Post2 period ( $x^{2}$ test $p$ $=0.019$ ), especially in older children, varying from $9.7 \%$ to $17.9 \%$ $\left(\mathrm{x}^{2}\right.$ test $\left.p=0.009\right)$. The prescription rates of co-amoxiclav did not change over periods, while cephalosporins prescription rates decreased significantly from $2 \%$ (Pre period) to $0.2 \%$ (Post2 period) (Fisher exact test $p=0.012$ ). Macrolides prescription rates varied from $12.6 \%$ to $16.4 \%$ to $7.3 \%$ in the Pre, Post 1 and Post2 periods with a significant ( $X^{2}$ test $p<0.001$ ) decrease in rates between the Post1 and Post2 periods. Regarding younger children, rates decreased significantly ( $x^{2}$ test $p=0.004$ ) from $20.7 \%$ (Post1 period) to $6.3 \%$ (Post2 period), similarly to children aged 4-6 months (from $16.4 \%$ to $7.7 \%, x^{2}$ test $p=0.042$ ) (Supplementary Table 1).

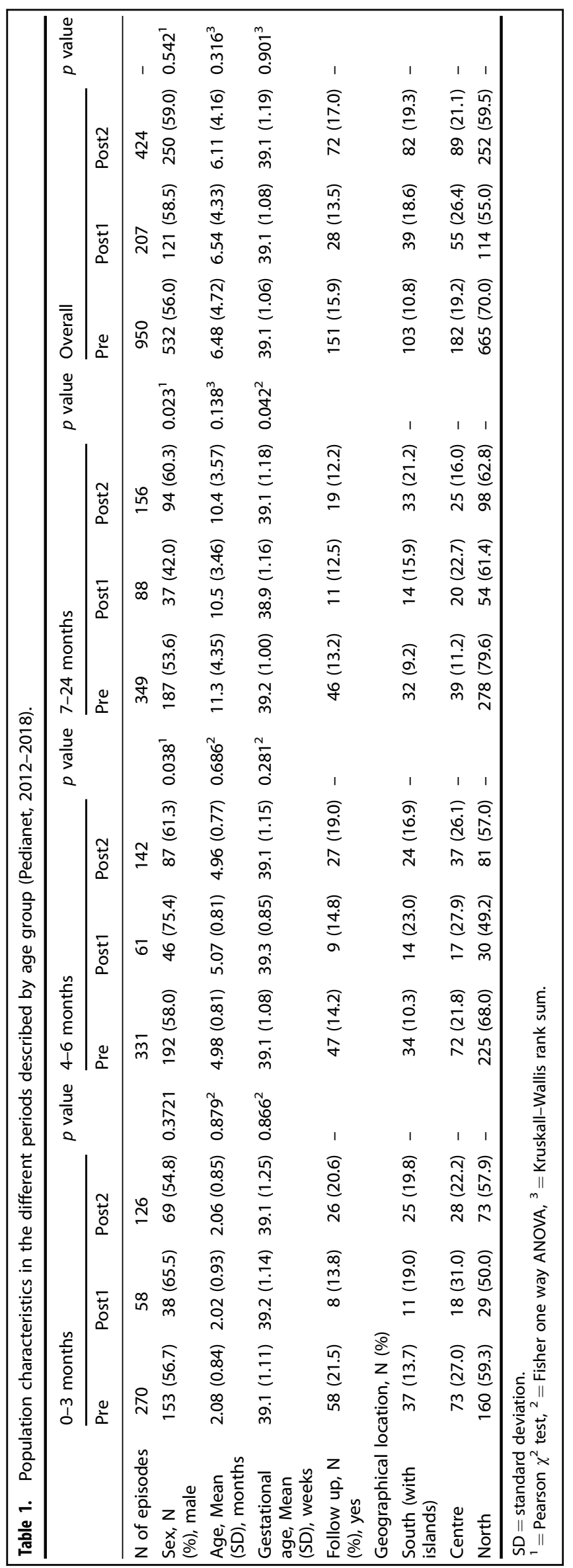



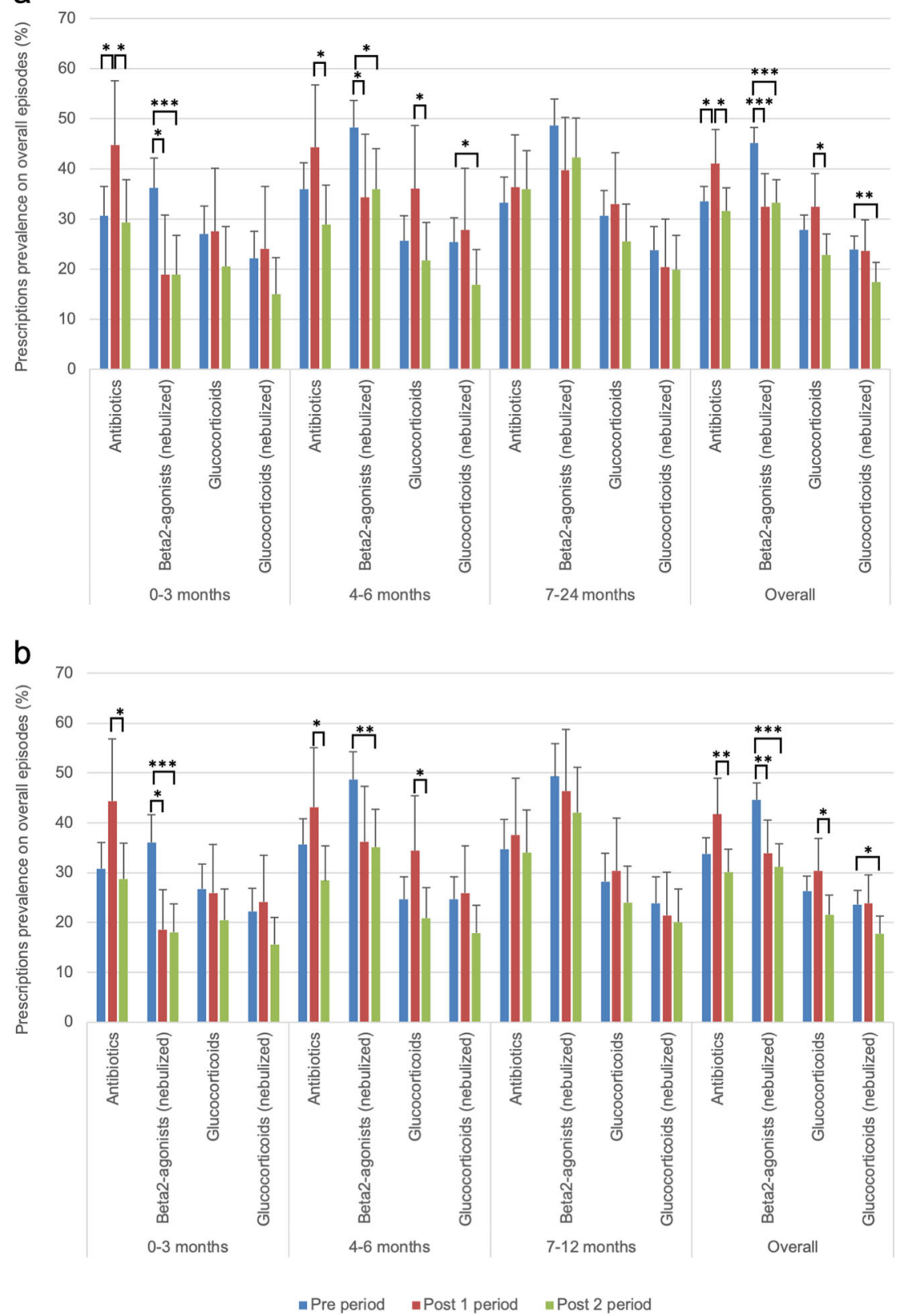

Fig. 2 Prevalence of treatments in the different periods and age groups (Pedianet, 2012-2018). Treatments for bronchiolitis episodes in the different periods described by age group in the overall cohort (a) and in children $<1$ year of age having a first episode of bronchiolitis (b). Positive error bars indicate the higher confidence interval limit (97.5\%) for the percentages. Significant $\chi^{2}$ test $p$ values are reported as asterisk $(*)$, if $0.05 \geq p$ value $>0.01$ then $*$, if $0.01 \geq p$ value $>0.001$ then ${ }^{* *}$, and if $p$ value $\geq 0.001$ then ${ }^{* *}$.

Nebulized $\beta 2$-agonists' prescriptions rates decreased relevantly in the Post 1 period from $45.1 \%$ to $32.4 \%$ ( $X^{2}$ test $p<0.001$ ) with stable rates in the Post2 period (33.3\%). Prescriptions rates dropped from $36.3 \%$ (Pre period) to $19.0 \%$ (Post1 and Post2 periods) in children younger than 3 months

The overall glucocorticoids prescription rates varied from $27.9 \%$ to $32.4 \%$ to $22.9 \%$ (Pre, Post1, Post2 periods). Nebulized glucocorticoids were prescribed in $23.9 \%$ of episodes in the Pre period and in the $17.5 \%$ of episodes in the Post2 period ( $x^{2}$ test $p=0.008)$; this reduction was particularly important in children aged 0-6 months (Fig. 2a).

Other respiratory drugs were prescribed in only 21 episodes, without changes between the analyzed periods (Supplementary Table 1).

\section{Post hoc analysis}

When considering the 1311 (82.9\%) first bronchiolitis episodes in children less than 1-year old, we obtained similar results. Overall antibiotics rate did not decrease after guidelines publication, but macrolides rates dropped from $11.8 \%$ in the Pre period to $7.6 \%$ in the Post2 period ( $X^{2}$ test $p=0.031$ ). Nebulized $\beta 2$-agonists' prescriptions rates (from $44.6 \%$ to $31.2 \%, x^{2}$ test $p<0.001$ ) and nebulized glucocorticoids significantly decreased (from $23.6 \%$ to $17.7 \%, \mathrm{X}^{2}$ test $p=0.024$ ) (Fig. $2 \mathrm{~b}$ and Supplementary Table 2).

\section{DISCUSSION}

According to our results, overprescribing is still very common for patients with bronchiolitis; the unnecessary use of medicine is 
even more alarming in the primary care context, where children are not presenting with severe symptoms and therefore medicine use is even less justified.

In the period analyzed, we did find a decrease in prescription rates: patients were prescribed medicines in the $66 \%$ of episodes before guidelines publication and in the $57 \%$ of episodes in the periods after. Even if this reduction was significant, it is clear that there is still much room for improvement.

In particular, antibiotic prescription rates did not change at all after the publication of both guidelines, with one third of the children diagnosed with bronchiolitis receiving at least one antibiotic. Bronchiolitis has a viral etiology, hence guidelines do not recommend antibiotics as first-line treatment; moreover, it has been demonstrated that overuse of antibiotics is related to resistant bacteria strains selection. Antibiotic misuse can be ascribed to the uncertainty in diagnosing bronchiolitis: differentiating it from bacterial pneumonia represents a challenge, especially in primary care, and pediatricians tend to treat the patient for both diseases ${ }^{23}$. This could be improved increasing rapid test utilization in PPC, such as RSV rapid test, since infants with RSV have low probabilities of having a serious bacterial infection $^{24,25}$, or procalcitonin and C-reactive protein rapid tests, which have been proven useful in differentiating viral and bacterial etiology in lower respiratory tract infections ${ }^{26}$. On the other hand, using rapid tests increase PPC-related costs, hence cost analysis is needed to determine the impact on the Italian healthcare system of extended rapid test usage.

We found a significant increase in amoxicillin prescriptions rates that could be ascribed to the publication of NICE guidelines for the treatment of pneumonia at the end of 2014, where amoxicillin was recommended as the first-line treatment for community-acquired pneumonia in children ${ }^{27}$. In PPC setting, IgM rapid tests or specific molecular diagnostic tests are scarcely used compared to the ED and hospital setting, thus the bacterial etiological suspect, once born, is rarely discarded. It should also be considered that the third dose to complete the recommended pneumococcal and Hib vaccination is delivered to the child at 11-12 months of age, thus family pediatricians might choose to prescribe a treatment covering a possible bacterial infection to younger children.

Another relevant finding was that children older than 7 months received more medicines than younger patients: after the publication of both bronchiolitis guidelines, in the $64.7 \%$ of episodes in children aged 7-24 months patients received pharmacological treatment, compared to the $51.9 \%$ in patients $<6$ months of age. This finding was coherent with the literature ${ }^{28}$; this difference could be explained by the fact that younger patients' bronchiolitis usually adheres better to the diagnostic criteria, while older patients are often diagnosed with bronchiolitis but treated for recurrent wheezing. However, when considering only the first bronchiolitis episode per child (thus excluding patients treated for recurrent wheezing), patients aged 7-24 months received more frequently a pharmacological treatment.

Numerous studies conducted mainly in the hospital setting ${ }^{29-38}$ evaluating the impact of bronchiolitis guidelines on medicine prescriptions reported that antibiotic prescription rates were the ones with a minor decrease among all treatments.

Focusing on the PPC, two studies, conducted in the same area of Spain, were particularly relevant: the first study reported a decrease in pharmacological treatment rates (from $72.5 \%$ to $52.1 \%)$ after a quality improvement initiative including interactive informal sessions, the distribution through the mail system of an evidence-based protocol and poster display in the ambulatory room to remind bronchiolitis diagnostic criteria and treatment recommendations ${ }^{29}$. In the second study, quality improvement initiatives were implemented both in PPC and in the corresponding hospital ED; the strategy included interactive educational sessions, bronchiolitis guideline algorithm distributed via emails, badges with the slogan "Bronchiolitis, less is more", informative posters in the waiting room, messages in report templates associated with the diagnostic code and monthly reports of performance ${ }^{39}$. With this intervention, they managed to reduce drastically the prescription of salbutamol (from $38.3 \%$ to $15.9 \%$ ), antibiotics (from $29.6 \%$ to $9.5 \%$ ) and corticosteroids (from $12.9 \%$ to $3.5 \%$ ). The Authors ascribe these results mainly to the collaboration between ED and family pediatricians; furthermore, having the project goal visible (i.e. in posters and badges) and giving the clinicians feedback on their prescription rates might have played a role in reducing the overuse of medications.

In another study conducted in a PPC clinic in the USA, the authors reported a significant reduction in beta2-agonists prescriptions rates in children with bronchiolitis from $45.7 \%$ to $13.7 \%{ }^{40}$. They combined guideline education for clinicians with a behavioral intervention approach based on findings revealing that being required to justify one's action causes more self-reflection, and that individuals prefer to conform to the actions of their peers ${ }^{41,42}$.

The reason for prescribing non-evidence-based treatments in acute bronchiolitis may vary: according to a British study, half of the general practitioners prescribed a treatment for a potential differential diagnosis, one third because a believed efficacy in the treatment and $31 \%$ because they believe that parents expect a prescription or that the prescription removes the need for a discussion with parents (it was possible for the clinician to choose more than one reason for prescribing medication) ${ }^{43}$. While the first reason could be valid-since it is challenging to differentiate between a clinical diagnosis of bronchiolitis, bronchopneumonia and recurrent wheeze-the others should raise some concerns because demonstrate a potential gap in the knowledge of bronchiolitis treatment, and a supposed negative influence of parents on clinical decisions.

This study is particularly relevant because it is the first study assessing prescriptions for bronchiolitis conducted in the Italian PPC setting and one of the few studies describing the impact of bronchiolitis guidelines on non-evidence-based treatments.

The strength of our study is its size, generalizability, and representative coverage of pediatric patients. In this study, $75 \%$ of the population referring to family pediatricians enrolled in Pedianet provided consent. In Italy, it is mandatory to be enrolled in primary care and children are assigned to their family pediatrician based on their home proximity to the family pediatrician ambulatory. Following this consideration, we have no evidence supporting a selection bias.

A limitation lies in its retrospective nature. It should not be excluded that at least a few bronchiolitis cases were seen in an ED without being reported to the family pediatricians. However, those would very likely have been identified because a follow-up examination by the family pediatrician is very always recommended after ED discharge, especially for younger children. Second, as per post hoc analysis, bronchiolitis diagnosis decreased in the years, possibly due to reduced misclassification. Indeed, the first part of both guidelines implemented was dedicated to the diagnosis, focusing on signs and symptoms. Bronchiolitis diagnoses were based on clinical evaluation, including those retrieved using International Classification of Diseases, Ninth Revision, Clinical Modification (ICD9-CM) codes assigned by the family pediatrician when reporting the physical evaluation in the patient's electronic medical chart in the software. Even if the dataset was manually validated, the impossibility to confirm clinical assessment is a well-recognized limitation in working with real-world data because it may be subjective to the attending clinician. We addressed this limit in case selection, applying an algorithm based on symptoms to the dataset. Third, it was not possible to evaluate prescription trend with a more sophisticated method such as regression analysis of interrupted time series because of the small sample size in monthly data for each 
outcome. Fourth, we chose to study just non-premature children with no comorbidities because of the regional differences in the reimbursement of palivizumab, a monoclonal antibody to prevent RSV infections, in respect of infants gestational age and comorbidities. Lastly, the impact of the guidelines was based on prescriptions and not on pharmacy dispensations, thus it was not possible to assess neither the "wait and see" approach (i.e. clinician could recommend the parents to wait $24-48 \mathrm{~h}$ before giving any medication to the child to allow the symptoms to improve spontaneously) neither the non-adherence.

In Italy, guidelines are mainly sponsored by scientific societies by publication on national and international peer-reviewed journals and emails. To change clinical practice, it is not enough for a guideline to be simply published and sent to health professionals: quality improvement represents valid strategies to decrease inappropriate prescribing ${ }^{44}$. The first step in this direction is represented by the campaign Choosing wisely, supported by the Italian Federation of Family Pediatricians that is implemented through clinicians' education and development of patient-friendly material about non-evidence-based treatments, with the slogan "Doing more does not mean doing better" ${ }^{45}$.

Stewardship policy implementation has also proved to make a difference in reducing inappropriate prescribing. In a setting where the main challenges are represented by high patient turnover rates and diagnostic uncertainty in empiric prescription, clinical pathways, a one-page decision support algorithm summarizing the guidelines, could be a valid tool to assist prescribers in defining the diagnosis and the most appropriate treatment ${ }^{46,47}$.

Non-evidence-based overprescribing of antibiotics and nebulized medication for infant bronchiolitis is still common.

For future research, we recommend a quality improvement strategy, targeting family pediatricians and ED clinicians and involving the same in the planning of the intervention, thus reducing in a more decisive way overtreatment and improving patients' outcomes.

\section{METHODS}

\section{Study design}

In this observational, uncontrolled before-after study, we assessed the changes in prescriptions for bronchiolitis in Italian PPC before (Pre period: December 2012-December 2014), in-between (Post1 period: December 2014-December 2015) and after (Post2 period: December 2015-December 2018) the publication of the two guidelines. The study flow chart is shown in Fig. 3.

\section{Data source}

Patients have been selected to review medical records collected by family pediatricians in the Pedianet Database. Pedianet is a PPC research database that collects information on children visited by 134 family pediatricians throughout Italy. The system is based on the transmission of specific data from computerized clinical files, that the pediatricians fill out during their daily clinical practice; informed consent is required from the parents ${ }^{48}$. Such data, generated using common software (JuniorBit ${ }^{\circ}$ ), are collected anonymously in a centralized database in Padua. The database contains several types of information, such as reason for the visit, medical examination, diagnosis, health status, prescriptions, specialist's referrals, hospitalizations, diagnostic procedures, growth parameters, and outcome data.

For this study data related to 150,018 children having at least 2 visits in the study period for a total of 2,958,459 visits were considered. Each family pediatrician had a median of 1156 (Interquartile range: 856-1886) patients registered in Pedianet in the study period with $54.5 \%$ of them residing in the North of Italy (Friuli-Venezia Giulia, Liguria, Lombardia, Piemonte, Veneto) and the rest in the Centre (15.7\%, Lazio, Marche, Toscana) and in the South or Islands (29.9\%, Abruzzo, Campania, Sardegna, Sicilia) according to ISTAT areas ${ }^{49}$.

The study and the access to the database were approved by the Internal Scientific Committee of Pedianet.

\section{Study population and case identification}

We included all children aged 0-24 months who were registered with one of the Pedianet family pediatricians since the birth between December 2012 and December 2018 and for whom parental informed consent was provided, with a diagnosis of acute bronchiolitis.

Cases have initially been identified from coded diagnosis of acute bronchiolitis (ICD9-CM codes 466.1, 466.11 or 466.19) and then from descriptive diagnosis in the free text fields. We also searched for patients with symptoms that can be ascribed to bronchiolitis; a case of acute bronchiolitis was defined as a first episode of respiratory distress combined with at least two of the following symptoms: coryza, cough, wheezing or crackles, tachypnea, chest retractions, skin color changes, nasal flaring, and fever $^{7,13}$. All the potential episodes were manually validated by two independent researchers (EB and SC) to exclude any false-positive cases. In case of disagreements, a consensus was reached with an expert pediatrician (DD).

In order to avoid duplicates, all visits occurring within 30 days of the initial diagnosis were considered as follow-up visits.

We considered the following as exclusion criteria: chronic complex conditions (such as cystic fibrosis, diabetes, chronic obstructive pulmonary disease), immunodeficiency or immunosuppressive therapy, prematurity ( $<37$ weeks of gestational age), Down syndrome, congenital heart disease other than small ventricular septal defect, hospitalization or visit in the ED in the 30 days before or after the diagnosis, concomitant bacterial infection (i.e. acute otitis media or pharyngotonsillitis) or ongoing antibiotic therapy (defined as an antibiotic prescription in the 14 days before the bronchiolitis case) and previous admission for wheeze to the ED.

\section{Outcomes}

The measured outcomes were the rates of prescriptions of oral systemic antibiotics, oral corticosteroids and nebulized corticosteroids, nebulized $\beta 2$-agonists, and other respiratory drugs identified with Anatomical Therapeutic Chemical codes (Supplementary Table 3).

We considered only the first prescription per drug class linked to the specific bronchiolitis episode.

\section{Statistical analysis}

Data analysis was performed on the whole cohort and then stratified based on age class (0-3, 4-6, and 7-24 months of age) basing the stratification in accordance with guidelines treatment indication. Demographic variables were compared among all periods and outcome variables were compared against all periods (Pre vs Post1, Pre vs Post2, and Post1 vs Post2). Post hoc

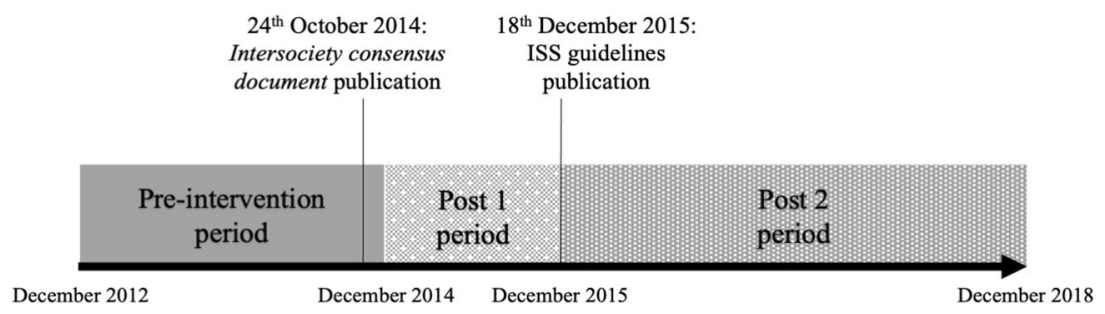

Fig. 3 Study design (Pedianet, 2012-2018). The arrow represents the timeline where are indicated the date of the publication of the two guidelines and the study periods with the corresponding starting and ending date colored with different textures of gray. ISS Istituto Superiore di Sanita' - Italian Institute of Health. 
analysis was performed considering just the first bronchiolitis episode per child and limiting the analysis to patient $<1$ year of age.

Categorical variables were expressed using frequencies and percentages; associations between these variables were assessed using the $x^{2}$ test (Pearson or Fisher exact test where appropriate).

Continuous variables were expressed as means and standard deviation (SD); these variables were compared using the appropriate non-paired test after assessing for the normality (Fisher one-way ANOVA or Kruskall-Wallis rank sum). The $95 \%$ confidence intervals were calculated with the Wilson Score method.

The analysis was performed using R statistical software- $\mathrm{v}$. 3.6.2 (Vienna, Austria $)^{50}$. Two sided $p<0.05$ was considered statistically significant.

\section{Reporting summary}

Further information on research design is available in the Nature Research Reporting Summary linked to this article.

\section{DATA AVAILABILITY}

The data used in this study cannot be made available in the manuscript, the supplemental files or in a public repository due to Italian data protection laws. The anonymized datasets generated during and/or analyzed during the current study can be provided on reasonable request, from the corresponding author, after written approval by the Internal Scientific Committee (info@pedianet.it).

\section{CODE AVAILABILITY}

Available upon request.

Received: 31 July 2020; Accepted: 17 February 2021; Published online: 19 March 2021

\section{REFERENCES}

1. Hall, C. B. et al. The burden of respiratory syncytial virus infection in young children. N. Engl. J. Med. 360, 588-598 (2009).

2. Pelletier, A. J., Mansbach, J. M. \& Camargo, C. A. Direct medical costs of bronchiolitis hospitalizations in the United States. Pediatrics 118, 2418-2423 (2006).

3. Deshpande, S. A. \& Northern, V. The clinical and health economic burden of respiratory syncytial virus disease among children under 2 years of age in a defined geographical area. Arch. Dis. Child 88, 1065-1069 (2003).

4. Subcommittee on Diagnosis and Management of Bronchiolitis. Diagnosis and management of bronchiolitis. Pediatrics. Pediatrics 118, 1774-1793 (2006).

5. Ralston, S. L. et al. Clinical practice guideline: the diagnosis, management, and prevention of bronchiolitis. Pediatrics 134, e1474-e1502 (2014).

6. National Institute for Health and Care Excellence. Bronchiolitis in children: diagnosis and management | Guidance | NICE. https://www.nice.org.uk/guidance/ng9 (2020). Accessed 20 Mar 2020.

7. Baraldi, E. et al. Inter-society consensus document on treatment and prevention of bronchiolitis in newborns and infants. Ital. J. Pediatr. 40, 65 (2014).

8. Gadomski, A. M. \& Scribani M. B. Bronchodilators for bronchiolitis. Cochrane Database Syst Rev. https://doi.org/10.1002/14651858.CD001266.pub4 (2014).

9. Hartling, L. et al. Epinephrine for bronchiolitis. Cochrane Database Syst Rev. https://doi.org/10.1002/14651858.CD003123.pub3 (2011).

10. Farley, R., Spurling, G. K., Eriksson, L. \& Mar, C. B. D. Antibiotics for bronchiolitis in children under two years of age. Cochrane Database Syst Rev. https://doi.org/ 10.1002/14651858.CD005189.pub4 (2014).

11. Fernandes, R. M. et al. Glucocorticoids for acute viral bronchiolitis in infants and young children. Cochrane Database Syst. Rev. https://doi.org/10.1002/14651858. CD004878.pub4 (2013).

12. Randolph, A. G. \& Wang, E. E. Ribavirin for respiratory syncytial virus lower respiratory tract infection. A systematic overview. Arch. Pediatr. Adolesc. Med. 150, 942-947 (1996).

13. Cartabellotta, A., Berlese, P. \& Drago, G. Linee guida per la diagnosi e il trattamento della bronchiolite nei bambini. Evidence 7, e1000128 (2015).

14. O'Brien, S. et al. Australasian bronchiolitis guideline. J. Paediatr. Child Health 55, 42-53 (2019).

15. Schuh, S. et al. Practice variation in acute bronchiolitis: a pediatric emergency research networks study. Pediatrics 140, e20170842 (2017).

16. Sacchetti, R. et al. Studio osservazionale multicentrico sulla bronchiolite nella Regione Emilia Romagna (SOMBRERO). Med. E Bambino 34, 376-381 (2015).
17. Jamal, A. et al. Pharmacotherapy in bronchiolitis at discharge from emergency departments within the Pediatric Emergency Research Networks: a retrospective analysis. Lancet Child Adolesc. Health 3, 539-547 (2019).

18. Korppi, M., Mecklin, M. \& Heikkilä, P. Review shows substantial variations in the use of medication for infant bronchiolitis between and within countries. Acta Paediatr. 108, 1016-1022 (2019).

19. Andrade, J. V., Vasconcelos, P., Campos, J. \& Camurça, T. Antibiotic prescribing in ambulatory care of pediatric patients with respiratory infections. Acta Med. Port. 32, 101-110 (2019).

20. Ochoa Sangrador, C. \& González de Dios, J. Research Group of the aBREVIADo Project. Overuse of bronchodilators and steroids in bronchiolitis of different severity: bronchiolitis-study of variability, appropriateness, and adequacy. Allergol. Immunopathol. (Madr.). 42, 307-315 (2014).

21. Zipursky, A. et al. International practice patterns of antibiotic therapy and laboratory testing in bronchiolitis. Pediatrics 146, e20193684 (2020).

22. Macias, C. G. et al. Variability in inpatient management of children hospitalized with bronchiolitis. Acad. Pediatr. 15, 69-76 (2015).

23. Ralston, S., Hill, V. \& Waters, A. Occult serious bacterial infection in infants younger than 60 to 90 days with bronchiolitis: a systematic review. Arch. Pediatr. Adolesc. Med. 165, 951-956 (2011).

24. Purcell, K. \& Fergie, J. Concurrent serious bacterial infections in 2396 infants and children hospitalized with respiratory syncytial virus lower respiratory tract infections. Arch. Pediatr. Adolesc. Med. 156, 322-324 (2002).

25. Purcell, K. \& Fergie, J. Concurrent serious bacterial infections in 912 infants and children hospitalized for treatment of respiratory syncytial virus lower respiratory tract infection. Pediatr. Infect. Dis. J. 23, 267-269 (2004).

26. Baumann, P. et al. Procalcitonin for diagnostics and treatment decisions in pediatric lower respiratory tract infections. Front Pediatr. 5, 183 (2017).

27. Pneumonia in adults: diagnosis and management | Guidance | NICE. https://www. nice.org.uk/guidance/cg191 (2020). Accessed 10 July (2020).

28. Montejo Fernández, M., Benito Manrique, I., Montiel Eguía, A. \& Benito Fernández, J. [An initiative to reduce the use of unnecessary medication in infants with bronchiolitis in primary care]. An. Pediatr. 90, 19-25 (2019).

29. Lopez, A. A., Aslanova, R., Bridger, N. \& Chafe, R. Antibiotic use for inpatient bronchiolitis: did national guidelines impact practice at a pediatric hospital? Hosp. Pediatr. 10, 147-152 (2020).

30. McCoy, E. \& Chumpia, M. Decreasing racemic epinephrine for bronchiolitis in an academic children's hospital. Hosp. Pediatr. 8, 651-657 (2018).

31. Parikh, K., Hall, M. \& Teach, S. J. Bronchiolitis management before and after the AAP guidelines. Pediatrics 133, e1-e7 (2014).

32. Akenroye, A. T., Baskin, M. N., Samnaliev, M. \& Stack, A. M. Impact of a bronchiolitis guideline on ED resource use and cost: a segmented time-series analysis. Pediatrics 133, e227-e234 (2014).

33. Ralston, S. L. et al. A multicenter collaborative to reduce unnecessary care in inpatient bronchiolitis. Pediatrics 137, e20150851 (2016).

34. Tejedor-Sojo, J. et al. Improving bronchiolitis care in outpatient settings across a health care system. Pediatr. Emerg. Care 35, 791-798 (2019).

35. Fontoura-Matias, J., Moreira-Sousa, D., Freitas, A. \& Azevedo, I. Management of bronchiolitis in Portugal, 2000-2015: Do guidelines have an impact? Pediatr. Pulmonol. 55, 198-205 (2020).

36. Henao-Villada, R., Sossa-Briceño, M. P. \& Rodríguez-Martínez, C. E. Impact of the implementation of an evidence-based guideline on diagnostic testing, management, and clinical outcomes for infants with bronchiolitis. Ther. Adv. Respir. Dis. 10, 425-434 (2016).

37. Gong, C., Byczkowski, T., McAneney, C., Goyal, M. K. \& Florin, T. A. Emergency department management of bronchiolitis in the United States. Pediatr. Emerg. Care 35, 323-329 (2019).

38. Walker, C., Danby, S. \& Turner, S. Impact of a bronchiolitis clinical care pathway on treatment and hospital stay. Eur. J. Pediatr. 171, 827-832 (2012).

39. Montejo, M. et al. Initiatives to reduce treatments in bronchiolitis in the emergency department and primary care. Arch Dis Child. https://doi.org/10.1136/ archdischild-2019-318085 (2019).

40. Sprecher, E. et al. Use of social psychology to improve adherence to national bronchiolitis guidelines. Pediatrics 143, e20174156 (2019).

41. Allison, S. T. \& Messick, D. M. The feature-positive effect, attitude strength, and degree of perceived consensus. Pers. Soc. Psychol. Bull. 14, 231-241 (1988).

42. Deutsch, M. \& Gerard, H. B. A study of normative and informational social influences upon individual judgement. J. Abnorm Psychol. 51, 629-636 (1955).

43. Carande, E. J., Cheung, C. R., Pollard, A. J. \& Drysdale, S. B. Change in viral bronchiolitis management in primary care in the UK after the publication of NICE guideline. Thorax 73, 674-676 (2018).

44. Powell, C. V. E. How to implement change in clinical practice. Paediatr. Respir. Rev. 4, 340-346 (2003).

45. Choosing Wisely Italia. Do not prescribe any drug (nebulized and/or by systemic route) for treatment of Bronchiolitis. Accessed May 25, 2020. https:// 
choosingwiselyitaly.org/en/raccomandazione-prof/do-not-prescribe-any-drugnebulized-and-or-by-systemic-route-for-treatment-of-bronchiolitis/ (2020). Accessed 25 May 2020.

46. Donà, D. et al. Effectiveness and sustainability of an antimicrobial stewardship program for perioperative prophylaxis in pediatric surgery. Pathogens 9, 490 (2020).

47. Donà, D. et al. Effects of clinical pathway implementation on antibiotic prescriptions for pediatric community-acquired pneumonia. PLOS ONE 13, e0193581 (2018).

48. Pedianet. https://pedianet.it/en/about (2020). Accessed 27 April 2020.

49. ISTAT. Glossario 2011. https://www.istat.it/it/files/2012/07/glossario_.pdf (2011). Accessed 7 July 2020.

50. R Core Team. R: A Language and Environment for Statistical Computing. https:// www.r-project.org/ (2020). Accessed 10 July 2020.

\section{ACKNOWLEDGEMENTS}

The authors thank all the family pediatricians collaborating in Pedianet. Angelo Alongi, Roberta Angelini, Giovanni Avarello, Lucia Azzoni, Maria Carolina Barbazza, Maria Barberi Frandanisa, Patrizia Barbieri, Gabriele Belluzzi, Eleonora Benetti, Roberto Bezzi, Franca Boe, Stefano Bollettini, Andrea Bruna, Ivana Brusaterra, Roberto Budassi, Massimo Caccini, Laura Cantalupi, Luigi Cantarutti, Luigia Caprio, Massimo Castaldo, Stefano Castelli, Serenella Castronuovo, Monica Cavedagni, Stefania Censini, Giuseppe Egidio Cera, Carla Ciscato, Mariangela Clerici Schoeller, Giuseppe Collacciani, Fabrizio Comaita, Ugo Alfredo Conte, Nicola Costanzo, Sandra Cozzani, Giancarlo Cuboni, Vito Francesco D'Amanti, Rita De Angelis, Roberto De Clara, Annamaria De Marchi, Emanuele De Nicolò, Gian Piero Del Bono, Gigliola Del Ponte, Tiziana Di Giampietro, Giuseppe Di Mauro, Giuseppe Di Santo, Piero Di Saverio, Marco Dolci, Mattia Doria, Stefano Drago, Pietro Falco, Mario Fama, Marco Faraci, Tania Favilli, Mariagrazia Federico, Michele Felice, Enrico Ferrara, Marta Ferrarese, Michele Ferretti, Paolo Forcina, Claudio Frattini, Ezio Frison, Fabrizio Fusco, Giovanni Gallo, Andrea Galvagno, Alberta Gentili, Pierfrancesco Gentilucci, Giuliana Giampaolo, Giuseppe Giancola, Silvia Girotto, Costantino Gobbi, Mauro Grelloni, Mirco Grugnetti, Urania Lagrasta, Massimo Landi, Paola Lasalvia, M.Rosaria Letta, Giuseppe Lietti, Cinzia Lista, Ricciardo Lucantonio, Francesco Luise, Diego Luotti, Nadia Macropodio, Francesca Marine, Lorenzo Mariniello, Gabriele Marostica, Sergio Masotti, Stefano Meneghetti, Massimo Milani, Stella Vittoria Milone, Angela Maria Monteleone, Pierangela Mussinu, Carmen Muzzolini, Flavia Nicoloso, Laura Olimpi, Maria Maddalena Palma, Vittorio Pandolfini, Angela Pasinato, Andrea Passarella, Pasquale Pazzola, Monica Perin, Danilo Perri, Silvana Pescosolido, Giovanni Petrazzuoli, Giuseppe Petrotto, Patrizia Picco, Ambrogina Pirola, Lorena Pisanello, Daniele Pittarello, Eleonora Polidoro, Elena Porro, Adolfo Francesco Porto, Elisabetta Profumo, Antonino Puma, Ferdinando Ragazzon, Paolo Rosas, Rino Rosignoli, Mariagiulia Rosina, Mariella Rossitto, Bruno Ruffato, Lucia Ruggieri, Annamaria Ruscitti, Annarita Russo, Pietro Salamone, Daniela Sambugaro, Luigi Saretta, Vittoria Sarno, Nico Maria Sciolla, Paolo Senesi, Carla Silvan, Valter Spanevello, Francesco Speranza, Maura Sticco, Francesco Storelli, Gianni Tamassia, Paolo Tambaro, Giacomo Toffol, Marco
Tondello, Gabriele Tonelli, Angelo Tummarello, Sergio Venditti, Concetta Volpe, Francescopaolo Volpe, Aldo Vozzi.

\section{AUTHOR CONTRIBUTIONS}

Conceptualization, E.B. and D.D.; methodology, E.B. and A:C.; software, E.B.; validation, E.B., A.C., D.D., and E.B.; formal analysis, E.B. and S.C.; investigation, E.B. and D.D.; resources, L.C. and C.G.; data curation, E.B. and SC.; writing-original draft preparation, S.C.; writing-review and editing, E.B., D.D, A.C., C.G, E.B., L.C.; visualization, E.B., A.C., and S.C.; supervision, D.D and C.G. All authors have read and agreed to the published version of the manuscript.

\section{COMPETING INTERESTS}

The authors declare no competing interests.

\section{ADDITIONAL INFORMATION}

Supplementary information The online version contains supplementary material available at https://doi.org/10.1038/s41533-021-00228-w.

Correspondence and requests for materials should be addressed to E.B.

Reprints and permission information is available at http://www.nature.com/ reprints

Publisher's note Springer Nature remains neutral with regard to jurisdictional claims in published maps and institutional affiliations.

\begin{abstract}
cc) (i) Open Access This article is licensed under a Creative Commons Attribution 4.0 International License, which permits use, sharing, adaptation, distribution and reproduction in any medium or format, as long as you give appropriate credit to the original author(s) and the source, provide a link to the Creative Commons license, and indicate if changes were made. The images or other third party material in this article are included in the article's Creative Commons license, unless indicated otherwise in a credit line to the material. If material is not included in the article's Creative Commons license and your intended use is not permitted by statutory regulation or exceeds the permitted use, you will need to obtain permission directly from the copyright holder. To view a copy of this license, visit http://creativecommons. org/licenses/by/4.0/.
\end{abstract}

C) The Author(s) 2021 